\title{
Hybrid Computational Intelligence Methods for Power System Optimization
}

\author{
Imran Rahman, Junita Mohamad-Saleh
}

\begin{abstract}
The best economic interest and arranging of electrical network framework has constantly worried an important worry within the power designing. constant improvement inside the petroleum by-product advent, large interconnection of the electrical structures and electricity emergency in the world require the monetary hobby of devoted strength delivering gadgets. subsequently, it is critical to contemplate most gifted streamlining strategies by way of getting basic elements of interest of simple detailing and execution of precise problem. This paper gives a diagram of large half of of and half of of computational knowledge (CI) techniques connected in power framework improvement. one-of-a-type applications and inspirations riding the enhancements of half of and $1 / 2$ CI techniques are accentuated. At final, some impending exploration regulations are proposed for the half of and 1/2 strategies improvement.
\end{abstract}

Keywords: Hybrid computational intelligence, power system, optimization, algorithm.

\section{INTRODUCTION}

Electricity framework enhancement has been a subject of massive research for state-of-the-art a long time, but the exam exertion modified into now and again moved to duplicate rehearses. The propensity has changed strongly inside the course of the most latest couple of years and energy framework development has now become a energetic place of research [1]. curiously, "Computational Intelligence (CI) is a computational manner to address cope with complex true issues to which numerical or regular demonstrating can be unrealistic for superb reasons just like the techniques can be unreasonably difficult for scientific questioning, it could contain a few vulnerabilities at some stage in the procedure, or the concept of the procedure may additionally additionally simply be stochastic" [2]. the second generation of enhancement calculations are issue express strategies and their usefulness is predicated upon the underlying supposition of the arrangement, so the ones systems (Like, GA, Simulated Annealing) likewise need problem particular computational plan. The 0.33 era calculations are perceived as advanced heuristic methods, transformative calculations or meta-heuristic. the essential thing wonderful of meta-heuristic strategies is set up strongly on irregular starting arrangements [3]. anyhow, the previously cited strategies extra frequently than now not revel in the sick outcomes of algorithmic unpredictability, premature combination and affectability to the underlying inquiry role

Revised Version Manuscript Received on August 19, 2019.

Imran Rahman, School of Electrical \& Electronic Engineering, Universiti Sains Malaysia (USM), Engineering Campus, Nibong Tebal, Pulau Pinang, Malaysia.

Junita Mohamad-Saleh, School of Electrical \& Electronic Engineering, Universiti Sains Malaysia (USM), Engineering Campus, Nibong Tebal, Pulau Pinang, Malaysia.
[1]. In a huge a part of the cases, first-rate effects are determined with the help of pass breed draws near, particularly on certifiable troubles [2]. anyways, in recent times, increasingly more collaboration conspires amongst preferred CI approach and accurate methodologies are proposed. these techniques more often than now not supply incredible results thinking about the reality that they can misuse the advantages of the two kinds of techniques on the equal time. it is critical to specify that, there may be a first rate prospect to research one-of-a-type bypass breed CI techniques for electricity framework enhancement and their related mind for future development [3].

In the the relaxation of this paper, creators will represent the half of and 1/2 Computational Intelligence (CI), past due types of hybridization procedures and utilization of go breed strategies in one-of-a-type electricity framework development troubles. At lengthy remaining, creators will show off a presentation research of overdue half of of breeds pursued with the aid of destiny hints and ends. really, it's miles almost tough to cover all half of breed CI strategies in a solitary article henceforth writers will accentuate on a few settled pass breed calculations identified with the most modern improvements.

\section{HYBRID TERMINOLOGY AND THEORY}

Half and 1/2 of CI techniques for the most part are at the least strategies that capacity jointly and offset every different to make a efficient cooperative power from their fuse. $1 / 2$ breed techniques play out a huge detail in improving the hunt capacity. All matters considered, the point of half of breed calculations is to join the upsides of each calculation at the equal time as trying to diminish any big shortcomings concurrently [3]. In a keynote discourse on computational information and building held in Istanbul, Turkey in the 12 months 2014, Professor Xin-She Yang recommended with recognize to the defenselessness of doing hybridization haphazardly [4]. assume, there exists $m$ sort of calculations, in the event that one alternatives to define a $1 / 2$ and half calculation, there will be ability mixes regular with the accompanying scenario [1]:

$$
C_{m}^{r}=\left(\begin{array}{l}
m \\
r
\end{array}\right)=\frac{m !}{r !(m-r) !}
$$

For $\mathrm{r}=3$ and $\mathrm{m}=10$, a hundred twenty mixtures, whilst for $r=2$ and $m=20$, there may be all out 190 half of of and halves, which may moreover deliver one hundred 90 strange 
(and almost futile) cross breeds. however, there can be shaped about $2 \mathrm{~m}=1,048,576$ half of of breed calculations by means of using allowing exquisite blends of $\mathrm{r}=2$, three... 19 . the entire of binomial coefficients is carried out for this depend. Analysts ought now not coin the calculation names to make half of breed. as an example, for any three calculations, as an instance, ACO, PSO and GA, any commonsense analysts ought now not create ACO-PSO-GA, or GA-PSO-ACO, or GAACOPSO. There want to be legitimate avocation earlier than doing any half of breed CI strategies.

\section{RECENT TRENDS ON HYBRID CI}

In a keynote discourse on computational understanding and designing held in Istanbul, Turkey inside the

The exam on combination CI techniques continues to be in its preliminary degree. anyways, professionals are propelled that, within the coming years, the majority of the logical productions on computational insight programs may be identified with hybridization [5]. however, the plan and execution technique of half of and half of of CI techniques is bit burdened and consists of mindfulness with respect to a enormous scope of algorithmic strategies, proper research, computational showing and genuinely as records systems. at some point of the time spent hybridization, it is extensive to painstakingly look into the contemporary written works at the fine improvement techniques to take care of associated troubles in reality due to the fact the investigation of diverse strategies for which include the most promising geologies of the perceived strategies. on this paper, the important problem is the half of and half CI techniques within the vicinity of improvement and electric powered framework. Fig. 1 presentations the every $\mathrm{yr}$ diaries round there from the 12 months 2004 till 2018.

\section{POWER SYSTEM OPTIMIZATION PROBLEMS}

Currently a-days, electric powered matrix framework holds an lively aspect at the identical time as in transit to the development of numerous divisions of any u . s . a .. A huge portion of the continuing problems associated with framework have non-curved and non-direct target works along overwhelming balance, imbalance imperatives and distinct styles of preference factors (regular, discrete and wide range) [6]. As current electric lattice frameworks come to be being an increasing number of thoughts boggling, appropriate interest, arranging and unique control of such frameworks utilising obsolete improvement techniques bear growing problems.

CI strategies can be powerful selections in mild of the ability to interrupt out network optima for tackling streamlining problems recognized with electricity framework [7]. In this text, writers examined written works depending at the $1 / 2$ of and $1 / 2$ strategies and energy framework streamlining. a part of the energy framework improvement zones are tested beneath.

Economic Dispatch. The time period 'financial dispatch' signifies the estimation of the overall power yield of the developing gadgets an awesome way to acquire the insignificant operating price [8].

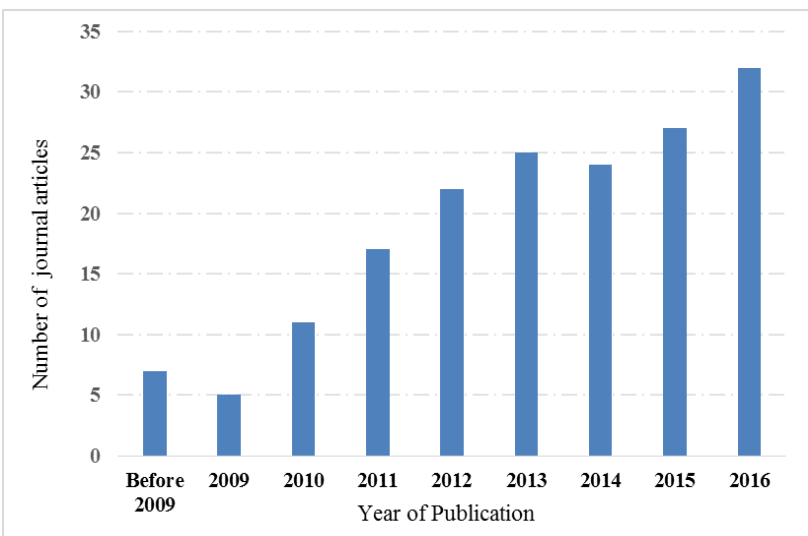

Fig. 1: Yearly data for research articles on optimization and power system

Pleasant energy go together with the flow (OPF). OPF characterizes as an unfaltering $\mathrm{u} . \mathrm{s}$. investigation below a given burden conditions wherein the thing is to examine the flows, voltages, responsive and actual power streams in a power framework and find out regular nation interest issue which limits age fee, misfortune, outflow and so forth [9]

Unit dedication (UC). UC alludes to the accumulation of the high-quality subset amongst numerous monetary reserving of making gadgets with a purpose to satisfy the heap request thru ostensible price uncovered to operating furthest reaches of age [10].

Burden Forecasting. Burden watching for is a approach used by the electricity companies for guaging the interest of intensity utilization in the end satisfy the need and deliver balance [7].

Generator upkeep Scheduling (GMS). GMS is an advancement trouble it really is non-at once, massive scale and stochastic in nature with specific clashing target capacities and requirements [11].

Dissemination Feeder Reconfiguration. The conveyance feeder reconfiguration hassle chooses on/off position of the strength dispersion device switches for proscribing force misfortune and in the long run sparing the rate [12].

Others. greater streamlining problems are deficiency finding, voltage soundness, delivered collectively power stream controller association, maximum intense loadability restrict and so forth.

within the present literary works, go breed CI techniques appear to be broadly settled. as an instance, turning into a member of Particle Swarm Optimization (PSO) with other single gadget appears, via all debts, to be fruitful in improving its presentation. by way of an extended shot, every the PSO bypass breeds and GA $1 / 2$ and halves are the maximum widely contemplated maximum of the analysts. proper here, Fig. 2 indicates research papers distributed (as indicated with the resource of SCOPUS database) recognized with above talked about territories. From the determine surely, mostapplied crossover strategies were inside the quarter of ideal power motion, economic dispatch and burden guaging streamlining. GA directors, as an instance, hybrid, alternate and desire had been consolidated into special 
techniques to get stepped forward up-and-comers [8].Except, $\mathrm{DE}, \mathrm{ACO}$ and moreover traditional network seek strategies had been used to do hybridization with PSO [8]. Albeit crossover $\mathrm{CI}$ techniques indicates outstanding gain of increasing population tremendous range and on this way enhancing the search ability of the propelled half of and half techniques, a few shortcomings do see on occasion. desk 1 demonstrates the usage of some ongoing $1 / 2$ and half $\mathrm{CI}$ strategies and offers remarks on their exhibition to looking after development troubles identified with electricity framework. In statistics to their observed articles, it thoroughly can be seen that most of the investigation in this calculation centered on algorithmic packages and alterations to manipulate framework streamlining, however there's still extensively greater hypothetical examinations need to do on this precise situation.

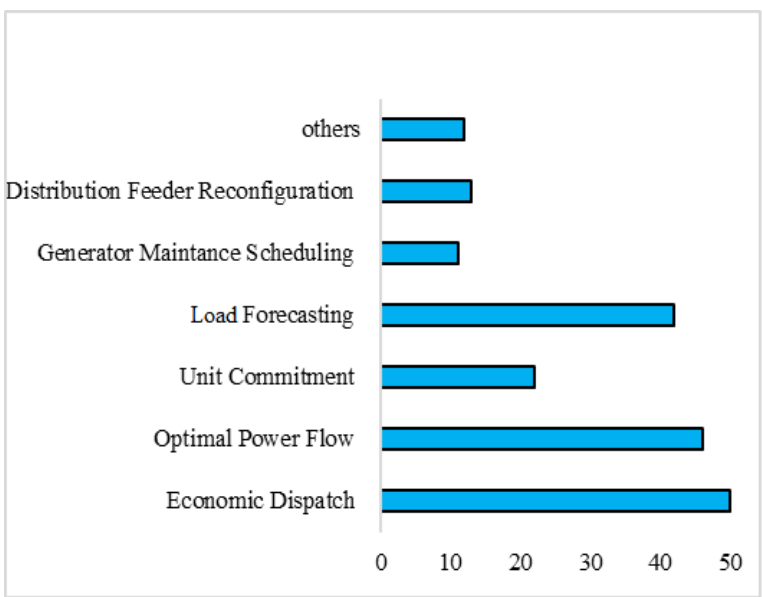

Fig. 2: Journal articles published on hybrid techniques and optimization of power system

Table 1: Application of hybrid CI methods for Power System Optimization

\begin{tabular}{l}
\hline Recent Hybrid CI methods \\
HPSO [8], CPSO-SQP [13], \\
FAPSO-NM [14], BFA-NM [15], \\
HSDE [16], CBPSO-RVM [17], \\
PSO-BFA [18], HGA-BFA [19], \\
FCASO [20], PSO-DE [21], GA-HS \\
[22], FFA-BA [23], MICA-IWO [24] \\
HICA-PS, HTSSA, DE-ABC, \\
ICA-PSO, HFPSO-NM, FFA-PS, \\
HMPSO, HNNBA, FWW [9] [25] \\
GA-DE [26], HTACS [27], AIS-GA \\
[28], PSO-GWO [29], PSO-GA [30]
\end{tabular}

PSO-LSSVM [31], PSO-QP [32], PSO-ACO [33], TV-SABC [34], FFA-SVR [35], Quantum PSO-SVR [36]

HHS [37], SA-ACO [38], HPSO [39], GA-IBPSO [40], TLBO-BHA [41], PSO-SFLA [42]

\section{Area in Power System Remarks}

Economic dispatch

Optimal Power Flow (OPF)

Unit Commitment (UC)

Load Forecasting

Generator

Scheduling

Maintenance

The hybrid PSO shows better result in terms of robustness and solution quality. Few hybrids ( BFA-PSO, HPSO) are computaionally cumbersome. Besides, there are few newly methods like BA-FFA and IWO- MICA exhibits better convergence.

In OPF problem, Hybrid PSO has also been used by most of the previous researchers. Both HMPSO and $\mathrm{DE}-\mathrm{ABC}$ shows better result in terms of computation time and accuracy.

Both hybrid GA and hybrid PSO methods improve the solution quality and convergence speed whereas hybrid HS algorithm shows less computation time for solving UC problems.

Hybrid PSO has been used mostly by the previous researchers for the optimization of load forecasting. Among all, PSO-ACO shows better convergence rate and robustness. Moreover, hybrid Quantum PSO provides accurate and interpretable forecasting.

Hybrid ACO technique is less sensitive to the control parameter variations. MTLBO-BH requires additional robust solution. Hybrid PSO-SFLA finds the best solution in terms of cost-effectiveness, robustness, reserve margin and computational complexity among the reviewed techniques.

Fuzzy-BA [43], HBB-BC [44], Distribution PSO-SFLA [45], PGSA-SaHDE [46], Reconfiguration

Feeder PSO-TS technique is both accurate and computationally efficient for solving particular problem whereas HBB-BC method enhances exploration capability with better converge rate.

PSO-BFOA shows robustness and effectiveness for optimal power system Stabilizers, both PSO-GA and PSO-DE show good convergence for estimating the maximum loadability limit of power systems. Moreover, HIA algorithm gives better minimum generation cost.
APSO-GA [48], PSO-GA [49], PSO-DE[50], PSO-BFOA [51], HIA Others [52] 


\section{V.FUTURE RESEARCH DIRECTIONS}

The Plausibility Of Choosing Unexplored Half Breed Strategies In Strength Framework Improvement Problems And Execute Them For The Examination Motive For Gift Is Extraordinarily An Appealing Area For Mainstream Researchers. Moreover, Diverse Written Works Have Through The Usage Of And Massive Clarified The Speculations At The Again Of These Half Of Of And 1/2 Of Strategies, And Except If Substantially New Discoveries Growth Out Internal The Ones Calculations, The Methodological Ramifications Might Be Of Lesser Enthusiasm Among Mainstream Researchers. All Topics Taken Into Consideration, It's Far Urging To Make Reference To That Thinking About The Fact That These Techniques Had Been Profoundly Used By Mainstream Researchers, They Are Organized For Appropriation By Manner Of And Via [48]. The Calculations Can Be Brazenly Embraced For Building Applications On The Grounds That Most People In Their Pseudo-Codes And Algorithmic Motive Has Merged And Institutionalized Over The Time Of Logical Studies. Matej Et Al. [53] Give Couple Of Rudimentary Rules To Play Out Any Examinations And Replications Of Ci-Based Streamlining Strategies. Moreover, Becoming Execution Measures Really As Proper Hugeness Test Should Be Completed For Honest Correlations.

\section{CONCLUSIONS}

This Section Condenses The Survey Of The Examinations On Cross Breed Ci Techniques As Given In Desk 1 In Which The Productions Identified With The Half Of Of And Half Of Of Ci Techniques Concerning Electricity Framework Utility, Alteration And Hybridization To Numerous Plans Of Combinatorial Enhancement Troubles Are Contemplated. It Very Well Can Be Visible That; The Improvement Of Half Of And 1/2 Calculations Is Expanding. Literary Works Have Validated That Very Little Works Have Been Completed On Hypothetical Part Of The Crossover Ci All In All, It Might Be Provocative To Play Out A Hypothetical Research Of The Heartiness And Assembly Houses Of This Calculation In Reality Because The Properly-Being Evaluation. The Plan Of Computationally Low Cost Cross Breed Calculations In Which There Will Be Much Less Parameters To Be Tuned Are Some Other Hypothetical Studies Territory This Is Deserving Of Exam. The design of computationally inexpensive hybrid algorithms where there will be less parameters to be tuned are another theoretical research area that is worthy of investigation.

\section{ACKNOWLEDGMENTS}

This research is supported by USM Global Fellowship (USM.IPS/USMGF/2/2016) and the Ministry of Higher Education (MOHE) Malaysia Fundamental Research Grant Scheme (Grant no. FRGS/1/2017/203.PELECT.6071371).

\section{REFERENCES}

1. T. Ting, X.- S. Yang, S. Cheng, And Okay. Huang, "Crossover Metaheuristic Calculations: Beyond, Gift, And Future," In Cutting-Edge Advances In Swarm Intelligence And Evolutionary Computation, Ed: Springer, 2015, Pp. 71-80 3.

2. E.- G. Talbi, "A Systematic Type Of Circulate Breed Metaheuristics," Magazine Of Heuristics, Vol. Eight, Pp.
541-564, 2002

3. Three. I. Rahman, P. Vasant, B. S. M. Singh, And M. Abdullah-Al-Wadud, "Swarm Intelligence-Based Totally Optimization For Phev Charging Stations," In Manual Of Research On Swarm Intelligence In Engineering, Ed, 2015, Pp. 374-405.

4. Four. Á. E. Eiben And M. Jelasity, "A Number One Be Conscious On Trial Studies Strategy In Ec," In Computational Intelligence, Court Instances Of The Area On Congress On, 2002, Pp. 582-587.

5. 5. I. Rahman And J. Mohamad-Saleh, "Module Electric Powered Powered Vehicle Charging Optimization The Use Of Bio-Stimulated Computational Intelligence Techniques," In Sustainable Interdependent Networks, Pp. 130 Five-147. Springer, Cham, 2018.

6. 6. L. Jourdan, M. Basseur, And E.- G. Talbi, "Hybridizing Correct Strategies And Metaheuristics: A Systematic Type," European Magazine Of Operational Studies, Vol. 199, Pp. 620-629, 2009.

7. 7. A. Askarzadeh, "Taking Care Of Electrical Energy Framework Troubles Through Amicability Seek: An Audit," Synthetic Intelligence Evaluation, Pp. 1-35, 2016.

8. 8. B. Kanna And S. N. Singh, "In The Direction Of Receptive Energy Dispatch Inside A Breeze Ranch Utilizing 1/2 Of And 1/2 Pso," Worldwide Magazine Of Electrical Electricity And Strength Systems, Vol. 69, Pp. 232-240, 2015.

9. Nine. A. L. A. Bolaji, M. A. Al-Betar, M. A. Awadallah, A. T Khader, And L. M. Abualigah, "A Miles Reaching Survey: Krill Herd Calculation (Kh) And Its Applications," Carried Out Tender Computing, Vol. Forty 9, Pp. 437-446, 2016.

10. 10. D. Bertsimas, E. Litvinov, X. A. Sun, J. Zhao, And T. Zheng, "Flexible Lively Enhancement For The Safety Obliged Unit Duty Hassle," Ieee Transactions On Energy Structures, Vol. 28, Pp. 52-Sixty Three, 2013.

11. Eleven. Ok. Dahal, K. Al-Arfaj, And K. Paudyal, "Showing Generator Aid Reserving Expenses In Deregulated Energy Markets," Ecu Magazine Of Operational Research, Vol. 240, Pp. 551-561, 2015.

12. 12. A. Kavousi-Fard And T. Niknam, "Best Dispersion Feeder Reconfiguration For Unwavering Fine Improvement Considering Vulnerability," Ieee Transactions On Electricity Transport, Vol. 29, Pp. 1344-1353, 2014

13. Thirteen. J. Cai, Q. Li, L. Li, H. Peng, And Y. Yang, "A Half Of Of And Half Of Cpso-Square Approach For Monetary Dispatch Thinking About The Valve-Factor Influences," Strength Conversion And Control, Vol. 53, Pp. One Hundred Seventy Five-181, 2012.

14. 14. T. Niknam, "Another Fluffy Flexible Flow Breed Molecule Swarm Improvement Calculation For Non-Direct, Non-Clean And Non-Curved Economic Dispatch Difficulty," Executed Energy, Vol. 87, Pp. 327-339, 2010

15. 15. R.- A. Hooshmand, M. Parastegari, And M. J. Morshed, "Emanation, Keep And Economic Burden Dispatch Problem With Non-Easy And Non-Arched Price Capacities Utilising The Skip Breed Bacterial Looking Nelder-Mead Calculation," Implemented Electricity, Vol. 89, Pp. 443-453, 2012.

16. 16. J. Zhang, Y. Wu, Y. Guo, B. Wang, H. Wang, And H. Liu, "A Half Of And Half Of Concordance Look Calculation With Differential Improvement For Day-Earlier Making Plans Issue Of A Microgrid With Notion Of Depth Circulation Imperatives," Applied Energy, Vol. 183, Pp. 791-804, 2016. 
17. 17. H. Lu, P. Sriyanyong, Y. H. Music, And T. Dillon, "Trial Investigation Of Some Other Half Of Breed Pso With Transformation For Monetary Dispatch With Non-Clean Fee Artwork," International Journal Of Electrical Electricity And Power Structures, Vol. 32, Pp. 921-935, 2010

18. 18. A. Y. Saber, "Financial Dispatch Utilising Molecule Swarm Development With Bacterial Looking Effect," International Mag Of Electrical Energy And Strength Structures, Vol. 34, Pp. 38-46, 2012.

19. 19. E. E. Elattar, "A Crossover Hereditary Calculation And Bacterial Scrounging Technique For Dynamic Economic Dispatch Problem," Global Mag Of Electrical Strength And Strength Systems, Vol. Sixty Nine, Pp. 18-26, 2015.

20. 20. J. Cai, Q. Li, L. Li, H. Peng, And Y. Yang, "A Fluffy Flexible Turbulent Insect Swarm Advancement For Monetary Dispatch," Global Magazine Of Electrical Energy And Electricity Systems, Vol. 34, Pp. 154-A Hundred And Sixty, 2012.

21.21. T. De Fátima Araújo And W. Uturbey, "Execution Evaluation Of Pso, De And 1/2 Of And Half Pso-De Calculations At The Same Time As Related To The Dispatch Of Age And Request," International Magazine Of Electrical Energy And Energy Structures, Vol. Forty Seven, Pp. 205-217, 2013

22. 22. S.- H. Huang And P.- C. Lin, "An Amicability Hereditary Primarily Based Absolutely Heuristic Approach Toward Financial Dispatching Consolidated Warmth And Electricity," Worldwide Journal Of Electrical Energy And Power Structures, Vol. Fifty Three, Pp. 482-487, 2013

23. 23. Y. A. Gherbi, H. Bouzeboudja, And F. Z. Gherbi, "The Joined Monetary Natural Dispatch Making Use Of New 1/2 Breed Metaheuristic," Power, Vol. One Hundred Fifteen, Pp. 468-477, 2016.

24. 24. M. Ghasemi, S. Ghavidel, M. M. Ghanbarian, And A. Habibi, "Each Different Aggregate Calculation For Nice Receptive Power Dispatch Difficulty With Discrete And Nonstop Manipulate Elements," Applied Smooth Computing, Vol. 22, Pp. 126-A Hundred And Forty, 2014

25. 25. N. Ming, W. Can, And X. Zhao, "A Survey On Utilizations Of Heuristic Streamlining Calculations For Ideal Strength Move In Current Power Frameworks," Magazine Of Current Electricity Structures And Clean Energy, Vol. 2, Pp. 289-297, 2014

26. 26. A. Trivedi, D. Srinivasan, S. Biswas, And T. Reindl, "A Hereditary Calculation Differential Improvement Based Totally Pass Breed Form: Case Check On Unit Obligation Making Plans Problem," Data Sciences, Vol. 354, Pp. 275-300, 2016.

27. 27. W. Yuan-Kang, H. Chih-Cheng, And L. Chun-Liang, "Goals Of The Unit Responsibility Problems By Means Of Using The 1/2 And Half Of Taguchi-Insect Agreement Framework Calculation," Worldwide Magazine Of Electrical Strength And Electricity Systems, Vol. Forty 9, Pp. 188-198, 2013

28. 28. Ok. Lakshmi And S. Vasantharathna, "Half Of Breed Faux Resistant Framework Technique For Benefit Based Totally Unit Responsibility Difficulty," Mag Of Electrical Engineering And Generation, Vol. Eight, Pp. 959-968, 2013

29. 29. V. Ok. Kamboj, "An Epic Half Of And 1/2 Of Pso-Gwo Method For Unit Obligation Problem," Neural Computing And Programs, Pp. 1-Thirteen, 2015

30. 30. N. Amjady And H. Nasiri-Rad, "Safety Obliged Unit Obligation By The Use Of Each Other Flexible Pass Breed Stochastic Pursuit Approach," Electricity Conversion And Manage, Vol. Fifty Two, Pp. 1097-1106, 2011

31. 31. Y. Chen, Y. Yang, C. Liu, C. Li, And L. Li, "A Move Breed Software Calculation Depending On The Assist Vector Device And Guy-Made Reasoning: A Case Of Electrical Burden Figuring Out," Implemented Mathematical Modeling, Vol. 39, Pp. 2617-2632, 2015.

32. 32. M. H. Moradi, M. Eskandari, And H. Showkati, "A 1/2 And 1/2 Of Approach For Synchronous Streamlining Of Dg Restrict And Operational System In Microgrids The Use Of Sustainable
Electricity Supply Property," Worldwide Journal Of Electrical Power And Energy Structures, Vol. Fifty Six, Pp. 241-258, 2014

33. 33. M. S. Kiran, E. Özceylan, M. Gündüz, And T. Paksoy, "An Epic Half Of Of And Half Method Depending On Molecule Swarm Streamlining And Subterranean Insect Province Calculation To Gauge Energy Request Of Turkey," Strength Conversion And Control, Vol. 53, Pp. Seventy Five-80 3, 2012

34. 34. N. Sulaiman, J. Mohamad-Saleh, And A. G. Abro, "Hearty Model Of Faux Honey Bee Kingdom (Ja-Abc4b) Calculation," Worldwide Magazine Of Bio-Stimulated Computation, Vol. 10, No. 2, Pp. 99-108, 2017.

35. 35. A. Kavousi-Fard, H. Samet, And F. Marzbani, "Any Other Half Of And Half Adjusted Firefly Calculation And Bolster Vector Relapse Version For Specific Temporary Burden Estimating," Expert Frameworks With Programs, Vol. Forty One, Pp. 6047-6056, 2014

36. 36. L.- L. Peng, G.- F. Fan, M.- L. Huang, And W.- C. Hong, "Hybridizing Demd And Quantum Pso With Svr In Electric Load Forecasting," Energies, Vol. Nine, P. 221, 2016

37. 37. M. Estahbanati, "Skip Breed Probabilistic-Congruity Search Calculation Method In Age Booking Problem," Mag Of Experimental And Theoretical Artificial Intelligence, Vol. 26, Pp. 283-296, 2014 